\title{
Reply to DM Hougaard et al.
}

\author{
Francisca Nordfalk $\mathbb{D}^{1} \cdot$ Claus Thorn Ekstrøm ${ }^{2}$
}

Received: 15 May 2019 / Accepted: 15 May 2019 / Published online: 28 June 2019

(c) The Author(s), under exclusive licence to European Society of Human Genetics 2019

We would like to thank Hougaard et al. [1] for the interest in our paper. As stated, the purpose of the paper was "to move beyond the primary screening purpose and elucidate how often and for which objectives the Danish NDBS samples are used for secondary purposes", as this has never been done before, neither by other researchers nor by the biobanks themselves. In the comment to the paper, Hougaard et al. state that we describe and criticize the usage of the Danish Newborn Screening Biobank (DNSB). We sincerely hope that readers of our paper will not interpret the paper as a criticism, but with our expressed goal in mind. Our paper clearly acknowledges how important a resource the Danish Newborn Screening Biobank is. Further, we believe that our efforts to elucidate the use of Danish newborn screening samples for research purposes will contribute to the justification of a state supported biobank like DNSB.

Hougaard et al. [1] express a wish to have seen our paper prior to publication. However, we tried several times to initiate a dialogue regarding the estimation of used samples and the number of samples in the biobank. As they were unable to meet our request and to provide us with relevant, detailed information, we got the firm impression that they were not interested in a collaboration about this topic.

In the comment by Hougaard et al. we identify two main areas of disagreement: the nature of the consent involved and the extent to which uses of samples are for secondary purposes. With respect to the nature of the

Francisca Nordfalk

nordfalk@sund.ku.dk

$\triangle$ Claus Thorn Ekstrøm

ekstrom@sund.ku.dk

1 Center for Medical Sciences and Technologies, Section for Health Services Research, Department of Public Health, University of Copenhagen, Copenhagen, Denmark

2 Section of Biostatistics, Department of Public Health, University of Copenhagen, Copenhagen, Denmark consent, however, it appears we are more or less in agreement, as we point out the legality, but also see a danger in lacking awareness of the donations in the general public. By the end of the comment, Hougaard et al. agree with the need for more public information to avoid misperceptions and distrust in the population. Indeed, this very correspondence acts as a step in the right direction, and we are therefore particularly pleased to see the response from Hougaard et al.

The second disagreement ostensibly raised in the comment by Hougaard et al. relates to our classification of 104 publications as secondary purposes and research. In their comment, Hougaard et al. state that 41 of the 104 publications should not be considered to have a secondary purpose as research, because they are either reporting of new screening programs, are projects screening for new diseases, or publications of technical methods. In our paper, we have thoroughly described and discussed the challenging distinction between what can be categorized as research and what is not. This difficulty has also been noted by other researchers as well as by the DNSB themselves in an earlier publication, as referred to in our paper. As to the comment regarding the usage of samples in studies of mental illness, we maintain our argument; that this is not related to the primary purpose of screening for mainly metabolic disorders. Hougaard et al. argue that the use of samples for studying mental disorders falls within newborn screening. However, the projects using the samples for these purposes are in every way considered research projects, which is also clearly stated in their publications. Considering for instance $\mathrm{iPSYCH}$, an almost $€ 50$ million research project, as a part of the Danish newborn screening program, is simply incorrect.

We were delighted to read how Hougaard et al. were now able to estimate how many of the DNSB samples that have been used for scientific projects. Considering the work, we have done, we are very much looking forward to the evidence supporting their numbers, as the DNSB are the only one who have access to the needed data. Moreover, 
we anticipate to study how Hougaard et al. have made the distinctions between what is considered screening, what is considered quality assurance and especially what they consider to be research based on the newborn screening samples.

We ended our paper with a hope that the results presented would contribute to discussions around the use of biological samples for research purposes. We view this correspondence as the first of hopefully many discussions on this topic. We are now even more optimistic that biobanks like the Danish Newborn Screening Biobank acknowledge the value of transparency in their work and an open debate with the research environment as well as with the general public.

\section{Compliance with ethical standards}

Conflict of interest The authors declare that they have no conflict of interest.

Publisher's note: Springer Nature remains neutral with regard to jurisdictional claims in published maps and institutional affiliations.

\section{References}

1. Hougaard DM, J Bybjerg-Grauholm, M Christiansen, B NørgaardPedersen. Response to "Newborn dried blood spot samples in Denmark: the hidden figures of secondary use and research participation”. Eu J Hum Genet. 2019. 